\title{
Role for Graduate Medical Education in the Response to COVID-19 Pandemic in Southeast Michigan Community Health Care System
}

\author{
Abdulghani Sankari ${ }^{1,2}$ Ali Zakaria1,๑ Glenn Taylor ${ }^{3,4,5}$ \\ ${ }^{1}$ Department of Medical Education, Ascension Providence Hospital, \\ Southfield, Michigan, United States \\ ${ }^{2}$ Department of Internal Medicine, Wayne State University, Detroit, \\ Michigan, United States \\ ${ }^{3}$ Department of Family Medicine, Ascension Providence Hospital, \\ Southfield, Michigan, United States \\ ${ }^{4}$ Michigan State University College of Human Medicine, Michigan, \\ United States \\ ${ }^{5}$ Michigan State University College of Osteopathic Medicine, \\ Michigan, United States
}

Address for correspondence Abdulghani Sankari, MD, PhD, 16001 West Nine Mile Rd., Southfield, MI 48075, United States (e-mail: Abdulghani.sankari@ascension.org).

\begin{abstract}
Keywords

- Graduate Medical Education

- COVID-19 pandemic

- trainee wellness

On March 10, 2020, the State of Michigan reported its first case of severe acute respiratory syndrome due to coronavirus disease 2019, which was admitted to Ascension Providence Hospital (APH). Michigan was the third most affected state in March 2020. To address the pandemic, Department of Graduate Medical Education joined the incident command team which consisted of APH leaders (Accreditation Council for Graduate Medical Education Designated Institutional Official, the Chief Medical Officer as commander, Chief Operating Officer, Chief of Logistics, Chief Nursing Officer, representatives from the medical and surgical sections, laboratory, finance, infection control, and occupational health). The team initiated the "crisis capacity surge plan" that was focused on patient care and led mainly by our trainee. In this correspondence we share our successful experience and provide our recommendation on how GME can navigate pandemic crisis.
\end{abstract}

\section{Introduction}

Ascension Providence Hospital (APH), Michigan, United States, is a 654-bed teaching complex located in the Metro Detroit Area. Graduate Medical Education (GME) in APH is well established with a total of 205 trainees across 18 specialties approved by the Accreditation Council for Graduate Medical Education (ACGME). To address the pandemic, we formed an incident command team consisted of APH leaders in collaboration with the Ascension Michigan State Incident Command Center. The critical

published online

August 4, 2021
DOI https://doi.org/

$10.1055 / \mathrm{s}-0041-1732282$

ISSN 2231-0770 need was in three main fronts: (1) emergency services, (2) intensive care units (ICUs), and (3) nonintensive care medical units.

To address the needs, we requested Pandemic Stage 3 emergency status from ACGME, ${ }^{1}$ which allowed the reassignment of residents to support these critical services under supervision. Fellows were divided into two groups: (1) eligible for appointment to emergency hospitalist and (2) noneligible who supported the "surge plan" under supervision. Trainees with higher risk (e.g., pregnancy or chronic illness) were assigned to telehealth ambulatory rotations. 


\section{The Development of Cohort Teams}

As the number of coronavirus disease 2019 (COVID-19) admissions increased while the number of other admissions steadily declined, patients with and without COVID-19 were interspersed throughout the hospital. This was accelerated once the Centers for Disease Control and Prevention (CDC) changed its recommendation to allow placing COVID-19 patients in regular hospital rooms with droplet precautions. $^{2}$

To care for these patients using "conventional capacity" standard infection control practices, the use of personal protective equipment (PPE) was expanding and the supply was limited. APH initiated the "crisis capacity surge plan" with implementation of COVID-19 cohort units. Under this plan, all COVID-19 patients were assigned to closed-units and were staffed by a predetermined group of caregivers: physicians, fellows, residents, and advanced practice providers. This accomplished three important needs: preservation of PPE by adapting prolonged use protocol per the CDC, ${ }^{2}$ standardization of care, and decrease risk of infection among health care workers.

Our experience was unique and the success of this model was due to: (1) immediate collaboration between GME and hospital to form incident command team; (2) forming closed-cohort units with predetermined team coverage; (3) multidisciplinary approach provided by physicians, nurses, respiratory therapists, social workers, and led by trainees; and (4) following the up-to-date guidelines on treatment of COVID-19 patients provided by APH and remaining on the units from 7 a.m. to 7 p.m. allowed standardization of care, prompt medical management of patients, and reduction in stress for other members of the team.

To ensure strict implementation of this model we had scheduled and organized rounds twice daily on every unit and direct communication with closed-cohort unit teams. All their concerns and feedback were documented and reported to the command center and adjustments were implemented immediately. This success was reflected by the positive feedback received from trainees participating in the model.

\section{Challenges in the Response to COVID-19 Pandemic at APH}

1. Shortage of PPE: APH faced a shortage of masks, respirators, and isolation gown.

2. Shortage of ventilators: APH incident command team reassigned ventilators from other Ascension hospitals. Also, anesthesia and noninvasive ventilators were repurposed, and used in operating rooms as ICU rooms. ${ }^{3}$ As a result, we avoided desperate measures such as dual-use of one ventilator for more than one patient, which has been discouraged by national guidelines. ${ }^{3}$

3. Burnout and wellness: GME and APH wellness team prepared a wellness plan to mitigate burnout as follows:

- Nutrition support including distribution of food.

- Provide clean and quiet rooms for trainees.
- Provide daily statistics on admissions and outcomes.

- Recognition of positive feedback on trainees.

- Response to trainees' concerns.

- Adequate "surge plan" schedule to maintain continuity of care.

- The mix among teams in each unit improved interpersonal communication skills and established rapport between trainees from different departments.

4. Education on COVID-19 and experimental therapies: GME, Simulation Center and Infection Control Department developed an up-to-date accessible online guideline for management of COVID-19. This included "Fundamentals of Critical Care Medicine" modules and in-house playbook for COVID-19 management.

5. Research related to COVID-19: GME initiated an expedited Ascension COVID-19 Data Registry Project and a coordinating center that included an epidemiologist, Designated Institutional Official (DIO), fellow, data manager, and director of scholarly activity to support trainees' scholarly activities.

6. Innovation in virtual care: Due to the high risk of exposure and limited PPE, virtual patient care became a necessity. The Association of American Medical Colleges has developed innovative open-access tools to learners as an alternative learning experience. ${ }^{4}$ Tablet computers were used for communication with patients admitted to COVID-19 units and for patients to communicate with their families. These devices were cleaned using well-defined infection-control procedures. ${ }^{5}$

\section{Recommendations for GME Programs}

- Identify early and request a Pandemic Stage 3 emergency status from ACGME. ${ }^{1}$

- Identify staffing needs.

- Identify high-risk trainees and assign to non-COVID-19 units.

- DIO to join the Incident Command center.

- Daily assessment of equipment needs and adequacy of supplies. ${ }^{6}$

- Provide Web-based training.

- Suspend elective procedures and redeploy trainees to crucial areas.

- Facilitate appointment of board-certified fellows as attendings in their core specialties. ${ }^{1}$

- Consider emergency funds for trainees.

- Celebrate trainees' accomplishments.

- Form a wellness plan for trainees including schedule a one-on-one meetings with the DIO.

- Utilize virtual learning and telemedicine.,7

\section{References}

1 Nasca TJ. ACGME's early adaptation to the COVID-19 pandemic: principles and lessons learned. J Grad Med Educ 2020;12(3):375-378 
2 Strategies for Optimizing the Supply of Isolation Gowns. Available at: https://www.cdc.gov/coronavirus/2019-ncov/ hcp/ppe-strategy/isolation-gowns. Accessed April 23, 2020

3 Aziz S, Arabi YM, Alhazzani W, et al. Managing ICU surge during the COVID-19 crisis: rapid guidelines. Intensive Care Med; 2020 ;46(7):1303-1325

4 Association of American Medical Colleges. iCollaborative. Clinical Teaching and Learning Experiences without Physical Patient Contact. Available at: https://icollaborative.aamc. org/collection/covid19-alternative-learning-experiences. Accessed June 9, 2021
5 Hollander JE, Carr BG. Virtually perfect? Telemedicine for Covid-19. Published March 11, 2020. Available at: https:// www.nejm.org/doi/full/10.1056/NEJMp2003539.Accessed June 9, 2021

6 Christian MD, Hawryluck L, Wax RS, et al. Development of a triage protocol for critical care during an influenza pandemic. CMAJ 2006;175(11):1377-1381

7 Woolliscroft JO. Innovation in response to the COVID-19 pandemic crisis. Acad Med 2020;95(8):1140-1142 